\title{
TEORES DE NUTRIENTES FOLIARES DE PESSEGUEIRO EM CINCO PORTA-ENXERTOS ${ }^{1}$
}

\author{
NEWTON ALEX MAYER ${ }^{2}$, BERNARDO UENO², VALÉCIA ADRIANA LUCAS DA SILVA ${ }^{3}$
}

RESUMO- Teores de nutrientes foliares indicam o estado nutricional da planta, auxiliam na recomendação de adubação e, em plantas frutíferas enxertadas, podem indicar combinações de copa/porta-enxerto incompatíveis. O objetivo do presente trabalho foi avaliar os teores de nutrientes foliares na cv. Maciel de pessegueiro, enxertada em cinco porta-enxertos ('Aldrighi', 'Capdeboscq', 'Flordaguard', 'Nemaguard' e 'Okinawa'). Dois pomares experimentais, localizados no município de Pelotas-RS, foram avaliados no $2^{\circ}$ e no $3^{\circ}$ anos após o plantio, respectivamente, nos anos de 2012 e 2013 . Conclui-se que os porta-enxertos 'Aldrighi', 'Capdeboscq', 'Flordaguard', 'Nemaguard' e 'Okinawa' não influenciaram nos teores foliares de P e de Mn da cv. Maciel, em ambos os pomares e anos de avaliação; o porta-enxerto 'Nemaguard' reduz os teores foliares de $\mathrm{Mg}$ da cv. Maciel, sem alterar a classe de interpretação agronômica; embora os teores foliares de N, Fe, Zn e B tenham sido abaixo do normal ou insuficientes, em praticamente todas as amostras de ambos os pomares e anos de avaliação, os porta-enxertos testados não influenciaram nestas interpretações. Termos para indexação: macronutrientes, micronutrientes, análise foliar, Prunus spp., Rosaceae.

\section{LEAF NUTRIENT CONTENT OF PEACH ON FIVE ROOTSTOCKS}

\begin{abstract}
Leaf nutrient content indicate the nutritional status of the tree, assist in fertilizer recommendation and may indicate incompatible scion/rootstock combinations in budded fruit trees. The objective of this study was to evaluate leaf nutrient contents of 'Maciel' peach budded on five rootstocks ('Aldrighi', 'Capdeboscq', 'Flordaguard', 'Nemaguard' and 'Okinawa'). Two experimental orchards located in Pelotas, Brazil, were evaluated on the $2^{\text {nd }}$ and $3^{\text {rd }}$ years after planting, respectively in 2012 and 2013. We concluded that 'Aldrighi', 'Capdeboscq', 'Flordaguard', 'Nemaguard' and 'Okinawa' rootstocks did not influence P and Mn leaf content on 'Maciel' peach scion, in both orchards and years of evaluation; 'Nemaguard' rootstock reduces Mg leaf content of 'Maciel' peach scion, but does not change the class of agronomic interpretation; although N, Fe, $\mathrm{Zn}$ and $\mathrm{B}$ leaf content were below than normal or insufficient in almost all samples from both orchards and years of evaluation, these studied rootstocks did not influence leaf nutrient interpretations.
\end{abstract}

Index terms: macronutrients, micronutrients, leaf analysis, Prunus spp., Rosaceae.

'(Trabalho 197-14). Recebido em:17-07-2014. Aceito para publicação em: 09-04-2015.

${ }_{2}^{2}$ Pesquisador da Embrapa Clima Temperado, BR 392, km 78, Caixa Postal 403, CEP 96010-971, Pelotas-RS. e-mail: alex.mayer@ embrapa.br; bernardo.ueno@embrapa.br

${ }^{3}$ Graduanda em Ciências Biológicas na Unviersidade Católica de Pelotas (UCPel), bolsista PIBIC/CNPq. e-mail: valecia_adriana@ hotmail.com 


\section{INTRODUÇÃO}

Estudos envolvendo nutrição em diferentes combinações copa e porta-enxerto auxiliam na recomendação de genótipos mais adequados às condições edafoclimáticas de uma região, na recomendação racional de fertilizantes, na redução de custos e na mortalidade de plantas, melhora a qualidade dos frutos, aumenta a produtividade e permite ampliar o cultivo para diferentes tipos de solo (TSIPOURIDIS et al., 2002; ROMBOLÀ et al., 2012). A avaliação de nutrientes nas folhas das copas também identifica a eficiência dos porta-enxertos na absorção e na translocação desses nutrientes, bem como os sintomas de incompatibilidade de enxertia entre os genótipos (REIGHARD et al., 2013).

A análise de solo não tem mostrado o método mais indicado para estimar-se a necessidade anual de alguns nutrientes em frutíferas lenhosas, como o potássio (FREIRE, 2002). Embora tenha sido proposta a análise química de ramos dormentes (JOHNSON et al., 2006) ou de flores (ZARROUK et al., 2005), a análise foliar de nutrientes em meados do verão é a prática padronizada e utilizada no mundo todo para determinar o estado nutricional das frutíferas (JOHNSON et al., 2006). A análise foliar de nutrientes, complementada com a expectativa de produtividade, a exportação de nutrientes pela poda verde, a poda de inverno e a queda das folhas permitem a reposição racional de nutrientes às plantas (TAGLIAVINI et al., 2000; FREIRE, 2002; SBCS/CQFS, 2004; ROMBOLÀ et al., 2012).

Em frutíferas de caroço, os teores de nutrientes foliares em plantas adultas variam em função da cultivar-copa, porta-enxerto, método de propagação (mudas enxertadas ou autoenraizadas), época de amostragem, idade da folha, carga produtiva da planta, ambiente pedoclimático e técnicas culturais aplicadas (COUVILLON, 1982; KNOWLES et al., 1984; BROWN; CUMMINS, 1989; BOYHAN et al., 1995; ZARROUK et al., 2005; LEONEL et al., 2011; ROMBOLÀ et al., 2012; REIGHARD et al., 2013). No Brasil, especialmente nos últimos 25 anos, diversos genótipos e cultivares têm sido estudados como porta-enxertos para frutíferas de caroço, em diferentes condições edafoclimáticas (CAMPO DALL'ORTO et al., 1992; DE ROSSI et al., 2004; MAYER et al., 2006; GALARÇA et al., 2012). Entretanto, informações sobre os efeitos dos porta-enxertos nos teores de nutrientes foliares são escassas na literatura brasileira.

O objetivo do presente estudo foi avaliar, no $2^{\circ}$ e no $3^{\circ}$ anos de idade, a influência de cinco porta-enxertos nos teores de nutrientes foliares da cv. Maciel de pessegueiro, em dois pomares experimentais.

\section{MATERIAL E MÉTODOS}

Mudas de pessegueiro [Prunus persica (L.) Batsch.] cv. Maciel (RASEIRA; NAKASU, 1998) foram produzidas em sacos plásticos $(28 \times 18 \mathrm{~cm})$ contendo substrato comercial. Para a produção dos porta-enxertos, utilizaram-se sementes estratificadas (PEREIRA; MAYER, 2005), oriundas de plantasmatrizes do Viveiro Frutplan Mudas Ltda. (PelotasRS). As cinco cultivares de porta-enxertos utilizadas foram: a) 'Aldrighi' (P. persica), cultivar selecionada na região de Pelotas durante a década de 1940 como produtora de frutos para indústria (RASEIRA; NAKASU, 1998). Essa cultivar também foi bastante utilizada como porta-enxerto no Sul do Brasil, até a década de 1970 (PEREIRA; MAYER, 2005); b) 'Capdeboscq' ( $P$. persica), cultivar-copa lançada em 1966 pela antiga Estação Experimental de Pelotas. Também foi bastante utilizada como porta-enxerto no Sul do Brasil, principalmente nas décadas de 1970 e 1980, em função, principalmente, da adaptação e da grande disponibilidade de suas sementes nas indústrias de conservas (PEREIRA; MAYER, 2005); c) 'Flordaguard', híbrido interespecífico lançado como porta-enxerto pela Universidade da Flórida (SHERMAN et al., 1991). Apresenta folhas vermelhas e é originário, em sexta geração, do cruzamento entre 'Chico 11' e Prunus davidiana (Carr.) Franch, C-26712.; d) 'Nemaguard' (P. persica), porta-enxerto americano selecionado em Fort Valley, estado da Geórgia (BROOKS; OLMO, 1961); e) 'Okinawa' $(P$. persica), originária do programa de Melhoramento Genético da Universidade da Flórida a partir de lote de sementes oriundas da ilha de Okinawa (Japão). É o porta-enxerto mais utilizado no Sudeste do Brasil para prunóideas (BARBOSA et al., 1993; PEREIRA; MAYER, 2005).

Em julho de 2010, as mudas foram implantadas em duas áreas experimentais, a saber: Pomar 1: área experimental da Sede da Embrapa Clima Temperado (3140’55,8”S; 52²6’7,39’O), com declividade do terreno para leste e altitude entre 54 e $56 \mathrm{~m}$. O espaçamento adotado foi de 6,5 m x 2 m (770 plantas ha-1). Pomar 2: área privada de um persicultor, com histórico da ocorrência de morte-precoce do pessegueiro, localizada na Colônia Júlio de Castilhos, $5^{\circ}$ distrito de Pelotas-RS (31³4’2,52”S; 52³0'23,71'O), com declividade do terreno para sudeste e altitude entre 127 e 132 m. O espaçamento utilizado foi de $5,7 \mathrm{~m}$ x $2 \mathrm{~m}$ (877 plantas $\left.\mathrm{ha}^{-1}\right)$. Em ambos os pomares, foram realizados 
os tratos culturais e as adubações recomendadas para a cultura do pessegueiro na região (RASEIRA et al., 1998; SBCS/CQFS, 2004). Em março de 2013, baseando-se nos resultados das análises de solo (Tabela 1), foi realizada a calagem no pomar 1, conforme recomendações da SBCS/CQFS (2004).

As amostragens de folhas, em ambos os pomares, foram feitas em 22-11-2012 e em 18-112013, ou seja, entre a 13a e a 15a semanas após a plena floração. Foram colhidas aproximadamente 100 folhas completas (limbo com pecíolo) ao redor das três plantas de cada parcela, na porção média dos ramos do ano localizados no terço mediano da planta, conforme as recomendações de Freire e Magnani (2005). As amostras foram acondicionadas em sacos de papel identificados e imediatamente enviadas ao Laboratório de Nutrição Vegetal da Embrapa Clima Temperado para análise química. Foram determinados os teores foliares dos macronutrientes nitrogênio $(\mathrm{N})$, fósforo $(\mathrm{P})$, potássio $(\mathrm{K})$, cálcio $(\mathrm{Ca})$ e magnésio $(\mathrm{Mg})$, expressos em \%, e dos micronutrientes ferro (Fe), manganês ( $\mathrm{Mn})$, zinco $(\mathrm{Zn})$, cobre ( $\mathrm{Cu})$ e boro (B), expressos em $\mathrm{mg}$ $\mathrm{kg}^{-1}$, de acordo com as metodologias definidas pela Rede Oficial de Laboratórios de Análise de Solo e de Tecido Vegetal do RS e de SC - ROLAS ( SBCS/ CQFS, 2004). Nas mesmas datas da amostragem das folhas, foram também coletadas amostras de solo (quatro subamostras por bloco, que formaram uma amostra composta por bloco), para avaliação do $\mathrm{pH}$, matéria orgânica (\%), P e K ( $\left.\mathrm{mg} \mathrm{dm}^{-3}\right), \mathrm{Ca}$ e $\mathrm{Mg}$ $\left(\mathrm{cmol}_{\mathrm{c}} \mathrm{dm}^{-3}\right)$, saturação $(\%)$ por bases e por alumínio e capacidade de troca de cátions $\left(\mathrm{CTC}_{\mathrm{pH}}{ }_{7}\right.$ ) (Tabela 1), para auxiliar na interpretação dos dados das análises foliares, conforme recomendação de Freire e Magnani (2005).

O delineamento experimental, em ambos os pomares, foi em blocos ao acaso, com cinco tratamentos (porta-enxertos 'Aldrighi', 'Capdeboscq', 'Flordaguard', 'Nemaguard' e 'Okinawa') e quatro repetições. Cada parcela foi constituída por três plantas, totalizando 60 plantas, em cada pomar. Os dados de cada pomar foram analisados separadamente e para cada ano de amostragem, sendo submetidos à análise de variância, pelo teste $\mathrm{F}$, e as médias comparadas pelo teste de Tukey, ao nível de $5 \%$ de probabilidade (ESTAT, 1994).

\section{RESULTADOS E DISCUSSÃO}

Os resultados das análises químicas de solo permitiram constatar a melhor condição de fertilidade no Pomar 2, em ambos os anos do estudo (Tabela
1). No Pomar 1, após amostragem do solo em 2012, realizou-se a calagem, conforme recomendações da SBCS/CQFS, (2004), o que reduziu sensivelmente a saturação por Al e aumentou o pH em água, no ano de 2013. Como destaque positivo em ambos os pomares, citam-se os níveis alto ou muito alto de $\mathrm{K}$ no solo e, como destaque negativo, os baixos ou médios teores de matéria orgânica (Tabela 1).

Os teores de macronutrientes foliares $(\mathrm{N}$, $\mathrm{P}, \mathrm{K}, \mathrm{Ca}$ e $\mathrm{Mg}$ ), avaliados em ambos os pomares experimentais, são apresentados na Tabela 2. No Pomar 1, observaram-se diferenças significativas entre os porta-enxertos testados somente para o N (2013) e para o Mg (ambos anos de avaliação), enquanto no Pomar 2, os efeitos significativos foram observados para o K (2012), Ca (2013) e $\mathrm{Mg}$ (ambos anos de avaliação). Portanto, para macronutrientes, o resultado mais consistente foi a redução dos níveis foliares de $\mathrm{Mg}$ nas plantas enxertadas sobre o 'Nemaguard'. Embora essa redução tenha sido estatisticamente significativa, não foi constatada mudança na faixa de interpretação dos níveis foliares de $\mathrm{Mg}$, pois todas as amostras dos diferentes porta-enxertos testados encontravam-se abaixo do normal ( Pomar 1) ou normal ( Pomar 2) (Tabela 2), de acordo com a classificação proposta pela SBCS/CQFS (2004). A redução dos teores foliares de $\mathrm{Mg}$ pelo porta-enxerto 'Nemaguard' parece depender da combinação com a cultivar-copa, pois Boyhan et al. (1995) também verificaram este efeito em ameixeiras (Prunus salicina Lindl.) cv. AU-Rosa, comparativamente às plantas enxertadas em 'Halford' e 'Lovell'. Entretanto, o efeito não foi significativo nas cultivares 'AU-Amber' e 'AUProducer'. Em pessegueiros das cultivares 'Loring' e 'Redhaven', o porta-enxerto 'Nemaguard' também reduziu os níveis foliares de $\mathrm{Mg}$ comparativamente a 'Lovell', 'Halford' e 'NC 152-AI-2' (KNOWLES et al., 1984). Na região sudeste dos Estados Unidos, 'Nemaguard' não é recomendado como porta-enxerto devido à sua baixa sobrevivência à morte-precoce (BOYHAN et al., 1995). O Mg é parte da molécula de clorofila, atua como ativador de diversas enzimas e correlaciona-se positivamente com o crescimento dos ramos (BOYHAN et al., 1995; JOHNSON, 2008).

Conforme estabelecido pela Sociedade (2004), os seguintes níveis de macronutrientes foliares são considerados normais para o pessegueiro e a nectarineira, nos Estados do Rio Grande do Sul e Santa Catarina: N (3,26-4,53 \%), P (0,15-0,28 \%), $\mathrm{K}(1,31-2,06 \%), \mathrm{Ca}(1,64-2,61 \%)$ e $\mathrm{Mg}(0,52-0,83$ $\%)$. No presente trabalho, segundo esta classificação, verificou-se que os níveis de $\mathrm{N}$ foliar, em ambos os pomares e anos de avaliação, foram abaixo do normal 
(exceção apenas para 'Nemaguard', no Pomar 1, em 2013), conforme ilustrado na Tabela 2.Outros elementos que também apresentaram níveis abaixo do normal foram o $\mathrm{Ca}$ e o $\mathrm{Mg}$, observado somente no Pomar 1, em todos os porta-enxertos testados e em ambos os anos de avaliação (Tabela 2). $\mathrm{O}$ pH do solo mais ácido no Pomar 1, além dos menores teores de Ca e Mg no solo, comparativamente ao observado no Pomar 2 (Tabela 1), foram as condições químicas do solo que contribuíram para estes menores níveis. Segundo Johnson (2008), o N é um nutriente que necessita ser aplicado em praticamente todos os pomares de pessegueiro nas diferentes regiões produtoras do mundo; é um elemento crítico para a vida da planta, compõe aminoácidos, proteínas, enzimas, ácidos nucleicos e clorofila. O Ca é o principal constituinte da parede e da membrana celular, e desempenha papel em seu funcionamento; também está envolvido na germinação do pólen, na divisão e na proteção celular. Deficiências de Ca geralmente não são problemas em pessegueiro (JOHNSON, 2008).

Para o K foliar, as análises de 2012 revelaram níveis abaixo do normal no Pomar 2, em todos os porta-enxertos, porém os níveis foram normais ou acima do normal no Pomar 1 (ambos anos) e também nas análises de 2013 no Pomar 2. De acordo com diversos levantamentos nutricionais realizados no Rio Grande do Sul, Freire (2002) verificou que, com base nas análises foliares em ameixeira, o potássio é um elemento que normalmente se encontra com teores normais ou acima do normal, sendo atribuídos, a este fato, a riqueza em $\mathrm{K}$ do material de origem dos solos gaúchos cultivados com a cultura e também o hábito dos fruticultores em utilizar formulações NPK nas adubações anuais.

Outro destaque positivo foi o $\mathrm{P}$, pois verificouse que todas as amostras, de todos os porta-enxertos, apresentaram níveis normal ou acima do normal, em ambos os anos avaliados (Tabela 2), evidenciando que o P não é elemento limitante ao desenvolvimento das plantas e à produção. Portanto, com base nas análises foliares realizadas, verificou-se que adubações fosfatadas ou formulações contendo P são desnecessárias para ambos os pomares estudados. De acordo com SBCS/CQFS (2004), não é observada resposta do pessegueiro à aplicação de fertilizante fosfatado na região Sul do Brasil, quando o teor de P foliar é maior do que $0,09 \%$. No presente estudo, constatou-se que, em ambos os pomares avaliados, os níveis de $\mathrm{P}$ foliar foram de 2,3 a 3,4 vezes maiores do que o limite mínimo.

Para os micronutrientes avaliados, os efeitos dos porta-enxertos testados não foram consistentes, considerando-se ambos os pomares e anos de avaliação. No Pomar 1, o 'Capdeboscq' reduziu os níveis de B (em 2012) e de Zn (em 2013), comparativamente ao 'Okinawa'. Estas diferenças não foram verificadas no Pomar 2 (Tabela 3). Por sua vez, no Pomar 2, verificou-se redução dos níveis foliares de $\mathrm{Cu}$ nas plantas enxertadas em 'Aldrighi', em ambos os anos de avaliação, comparativamente às plantas enxertadas em 'Flordaguard'. Contudo, estas diferenças não foram verificadas no Pomar 1. Os dados de 2012, no Pomar 2, revelam que 'Nemaguard' reduziu o teor foliar de Fe $(72,5 \mathrm{mg}$ $\left.\mathrm{kg}^{-1}\right)$, comparativamente ao 'Capdeboscq' (88,8 mg $\mathrm{kg}^{-1}$ ) (Tabela 3). Este resultado pode estar associado ao $\mathrm{pH}$ mais elevado do solo $(7,0)$ (Tabela 1 ), quando o Fe pode tornar-se imobilizado dentro da planta, ocasionando a clorose férrica (JOHNSON, 2008). Em solos alcalinos, o 'Nemaguard' é um dos porta-enxertos mais suscetíveis à clorose férrica (ROMERA et al., 1991).

Para micronutrientes, os seguintes valores são considerados normais para análises foliares em pessegueiro e nectarineira ( $\mathrm{SBCS} / \mathrm{CQFS}, 2004)$ : B (34-63 mg kg-1), Cu (6-30 mg kg-1), Fe (100-230 $\left.\mathrm{mg} \mathrm{kg}{ }^{-1}\right), \mathrm{Mn}$ (31-160 mg kg-1) e Zn (24-37 mg $\left.\mathrm{kg}^{-1}\right)$. De acordo com esta classificação, constatase que praticamente todas as amostras dos cinco porta-enxertos testados apresentaram níveis de $\mathrm{Mn}$ e $\mathrm{Cu}$ na faixa normal, com exceção apenas das amostras provenientes do Pomar 2 no ano de 2013, classificadas como abaixo do normal (Tabela 3). Para os micronutrientes avaliados no presente estudo, nenhuma das amostras foi classificada nas faixas acima do normal ou excessivo. Os níveis de Fe, Zn e B encontraram-se abaixo do normal ou insuficientes, em praticamente todas as amostras provenientes de ambos os pomares e anos de avaliação, conforme legenda inserida na Tabela 3. Constata-se, novamente, que os porta-enxertos testados não alteraram, de forma consistente, as faixas de interpretação dos níveis de micronutrientes avaliados. Segundo Johnson (2008), o principal efeito do Fe na planta é a transferência de energia durante o processo de fotossíntese e respiração; a deficiência de Fe é o principal problema nutricional em regiões persícolas com solos alcalinos ( $\mathrm{pH}$ entre 7,5 e 8,5). O Zn atua na formação das auxinas, e sua falta causa a desordem conhecida como "folha pequena", encontrada em quase todas as regiões produtoras mundiais. Já o B é nutriente essencial para o crescimento e desenvolvimento da planta; atua no crescimento do tubo polínico e do meristema, transporte de açúcares, síntese da parede celular, produção de hormônios e integridade da membrana. Em nível mundial, deficiências de B não são comuns em pessegueiro (JOHNSON, 2008). 
Os resultados do presente trabalho reforçam a importância de se ter conhecimento das cultivares utilizadas como porta-enxertos em pessegueiro. Adicionalmente, a produção das mudas em embalagens plásticas com substrato parece ter importância decisiva na sobrevivência inicial no campo, devido à manutenção do torrão que envolve as raízes e a preservação de suas radicelas no transplantio, visto que não ocorreu nenhuma morte de plantas, em ambos os experimentos. Em área imediatamente adjacente ao Pomar 2, implantada em 2010 com mudas adquiridas no comércio local, de raiz nua da cv. Maciel com uso de porta-enxertos desconhecidos (misturas de caroços provenientes do resíduo de indústria processadora), a mortalidade de plantas foi de $37 \%$ até 2013, algumas inclusive devido à morte-precoce do pessegueiro.

TABELA 1- Resultados e interpretação da análise de solo dos dois pomares experimentais, em 2012 e $2013^{1}$. Embrapa Clima Temperado, Pelotas-RS.

\begin{tabular}{|c|c|c|c|c|c|c|c|c|c|c|c|c|c|}
\hline \multirow{2}{*}{ Ano } & \multirow{2}{*}{$\begin{array}{l}\mathrm{pH}_{\text {agua }} \\
1: 1 \\
\end{array}$} & \multirow{2}{*}{$\begin{array}{l}\text { M.O. } \\
(\%)\end{array}$} & \multirow{2}{*}{\multicolumn{2}{|c|}{$\frac{\mathrm{P} \quad \mathrm{K}}{\mathrm{mg} \mathrm{dm^{-3 }}}$}} & \multirow[t]{2}{*}{$\mathrm{Na}$} & \multirow{2}{*}{$\mathrm{Ca}$} & \multirow{2}{*}{\multicolumn{3}{|c|}{$\frac{\mathrm{Mg} \mathrm{Al} \mathrm{H}+\mathrm{Al}}{\mathrm{cmol} \mathrm{dm}^{-3}}$}} & \multirow[t]{2}{*}{$\mathrm{K}$} & \multicolumn{2}{|c|}{ Saturação (\%) } & \multirow{2}{*}{$\begin{array}{l}\mathrm{CTC} \\
\mathrm{pH} 7\end{array}$} \\
\hline & & & & & & & & & & & $\mathrm{Al}$ & Bases & \\
\hline \multicolumn{14}{|c|}{ Pomar 01} \\
\hline 2012 & $\begin{array}{l}5,0 \\
(\mathrm{Mb})\end{array}$ & $\begin{array}{l}1,5 \\
\text { (B) }\end{array}$ & $\begin{array}{c}4,7 \\
(\mathrm{Mb})\end{array}$ & $\begin{array}{l}84 \\
(\mathrm{~A})\end{array}$ & 12 & $\begin{array}{l}1,3 \\
\text { (B) }\end{array}$ & $\begin{array}{l}0,7 \\
(\mathrm{M})\end{array}$ & 0,5 & 4,5 & 0,2 & $\begin{array}{l}20,2 \\
(\mathrm{~A})\end{array}$ & $\begin{array}{c}33 \\
(\mathrm{Mb})\end{array}$ & $\begin{array}{l}6,7 \\
(\mathrm{M})\end{array}$ \\
\hline 2013 & $\begin{array}{l}5,6 \\
(\mathrm{M}) \\
\end{array}$ & $\begin{array}{l}1,1 \\
\text { (B) }\end{array}$ & $\begin{array}{l}8,7 \\
(\mathrm{~B})\end{array}$ & $\begin{array}{l}90 \\
(\mathrm{~A})\end{array}$ & 15 & $\begin{array}{l}1,6 \\
\text { (B) }\end{array}$ & $\begin{array}{l}0,8 \\
(\mathrm{M})\end{array}$ & 0,2 & 1,6 & 0,2 & $\begin{array}{l}7,2 \\
(\mathrm{~B})\end{array}$ & $\begin{array}{l}60 \\
\text { (B) }\end{array}$ & $\begin{array}{l}4,2 \\
(\mathrm{~B})\end{array}$ \\
\hline \multicolumn{14}{|c|}{ Pomar 02} \\
\hline 2012 & $\begin{array}{l}7,0 \\
\text { (A) }\end{array}$ & $\begin{array}{l}2,7 \\
(\mathrm{M})\end{array}$ & $\begin{array}{l}30,2 \\
(\mathrm{~A})\end{array}$ & $\begin{array}{l}81 \\
(\mathrm{~A})\end{array}$ & 19 & $\begin{array}{l}7,2 \\
\text { (A) }\end{array}$ & $\begin{array}{l}2,4 \\
\text { (A) }\end{array}$ & 0,0 & 1,5 & 0,2 & $\begin{array}{c}0,0 \\
(\mathrm{Mb})\end{array}$ & $\begin{array}{l}87 \\
\text { (A) }\end{array}$ & $\begin{array}{l}11,3 \\
(\mathrm{M})\end{array}$ \\
\hline 2013 & $\begin{array}{l}6,8 \\
\text { (A) }\end{array}$ & $\begin{array}{l}1,8 \\
(\mathrm{~B})\end{array}$ & $\begin{array}{l}56,8 \\
(\mathrm{Ma})\end{array}$ & $\begin{array}{c}139 \\
(\mathrm{Ma})\end{array}$ & 24 & $\begin{array}{l}4,9 \\
\text { (A) }\end{array}$ & $\begin{array}{l}1,7 \\
\text { (A) }\end{array}$ & 0,0 & 1,0 & 0,4 & $\begin{array}{c}0,0 \\
(\mathrm{Mb})\end{array}$ & $\begin{array}{l}88 \\
\text { (A) }\end{array}$ & $\begin{array}{l}8,1 \\
(\mathrm{M})\end{array}$ \\
\hline
\end{tabular}

${ }^{1}$ Média das análises provenientes dos quatro blocos experimentais. Interpretação das análises químicas (SOCIEDADE, 2004): Mb = muito baixo; $\mathrm{B}=$ baixo; $\mathrm{M}=$ médio; $\mathrm{A}=$ alto; $\mathrm{Ma}=$ muito alto.

TABELA 2- Conteúdo de macronutrientes nas folhas (em \%) da cv. Maciel de pessegueiro,em diferentes porta-enxertos, em dois pomares experimentais. Embrapa Clima Temperado, 2012-2013.

\begin{tabular}{|c|c|c|c|c|c|c|c|c|c|c|}
\hline \multirow{2}{*}{$\begin{array}{l}\text { Porta- } \\
\text { enxerto }\end{array}$} & & V. & & & & & & & & \\
\hline & 2012 & 2013 & 2012 & 2013 & 2012 & 2013 & 2012 & 2013 & 2012 & 2013 \\
\hline & \multicolumn{10}{|c|}{ Pomar 1} \\
\hline Aldrighi & $72 a$ & $3,16 \mathrm{~b}$ & $0,21 \mathrm{a}$ & $0,31 \mathrm{a}$ & $2,02 \mathrm{a}$ & $2,53 \mathrm{a}$ & $1,23 \mathrm{a}$ & $1,41 \mathrm{a}$ &, $41 a b$ & $0,51 \mathrm{a}$ \\
\hline Capdeboscq & 57 a & $3,15 \mathrm{~b}$ & $0,21 \mathrm{a}$ & $0,27 \mathrm{a}$ & $2,08 \mathrm{a}$ & $2,39 \mathrm{a}$ & $52 \mathrm{a}$ & $61 \mathrm{a}$ & b & $0,51 \mathrm{a}$ \\
\hline Flordaguard & $2,74 \mathrm{a}$ & $3,21 \mathrm{~b}$ & $0,24 \mathrm{a}$ & $0,31 \mathrm{a}$ & $2,05 \mathrm{a}$ & $2,40 \mathrm{a}$ & $1,38 \mathrm{a}$ & $1,62 \mathrm{a}$ & ,44 a & $0,51 \mathrm{a}$ \\
\hline Nemaguard & $2,68 \mathrm{a}$ & $3,50 \mathrm{a}$ & $0,24 \mathrm{a}$ & $0,31 \mathrm{a}$ & $2,06 \mathrm{a}$ & $2,49 \mathrm{a}$ & $1,34 \mathrm{a}$ & $1,32 \mathrm{a}$ & $37 \mathrm{~b}$ & $0,42 \mathrm{~b}$ \\
\hline Okinawa & $2,61 \mathrm{a}$ & $3,24 \mathrm{ab}$ & $0,26 \mathrm{a}$ & $0,30 \mathrm{a}$ & $2,19 \mathrm{a}$ & $2,44 \mathrm{a}$ & $1,38 \mathrm{a}$ & $1,37 \mathrm{a}$ & $0,40 \mathrm{ab}$ & $0,46 \mathrm{ab}$ \\
\hline $\mathrm{F}$ & $0,34^{\mathrm{ns}}$ & $5,68^{* *}$ & $1,81^{\mathrm{ns}}$ & $1,17^{\mathrm{ns}}$ & $0,60^{\mathrm{ns}}$ & $0,69^{\mathrm{ns}}$ & $1,70^{\mathrm{ns}}$ & $3,98^{*}$ & $5,61^{* *}$ & $9,12^{* *}$ \\
\hline$F_{1}$ & $0,85^{\text {ns }}$ & $2,00^{\text {ns }}$ & $2,38^{\text {ns }}$ & $1,57^{\mathrm{ns}}$ & $1,71^{\mathrm{ns}}$ & $0,04^{\mathrm{ns}}$ & $1,07^{\mathrm{ns}}$ & $9,07^{* *}$ & $10,76^{* *}$ & $10,39^{* *}$ \\
\hline \multirow[t]{2}{*}{ CV (\%) } & 20 & 72 & 14,46 & 60 & 33 & 5,54 & 11,64 & 9,53 & 20 & 5,76 \\
\hline & \multicolumn{10}{|c|}{ Pomar 2} \\
\hline Aldrighi & $2,71 \mathrm{a}$ & $2,92 \mathrm{a}$ & $0,25 \mathrm{a}$ & $0,25 \mathrm{a}$ & $1,12 \mathrm{ab}$ & $2,23 \mathrm{a}$ & $2,42 \mathrm{a}$ & $2,46 \mathrm{a}$ &, $72 \mathrm{a}$ & $0,78 \mathrm{a}$ \\
\hline Capdeboscq & $2,82 \mathrm{a}$ & $3,08 \mathrm{a}$ & $0,28 \mathrm{a}$ & $0,27 \mathrm{a}$ & $1,12 \mathrm{ab}$ & $2,05 \mathrm{a}$ & $2,43 \mathrm{a}$ & $2,54 \mathrm{a}$ & $0,68 \mathrm{a}$ & $0,75 \mathrm{a}$ \\
\hline Flordaguard & $2,82 \mathrm{a}$ & $2,95 \mathrm{a}$ & $0,30 \mathrm{a}$ & $0,27 \mathrm{a}$ & $1,11 \mathrm{ab}$ & $2,00 \mathrm{a}$ & $2,54 \mathrm{a}$ & $2,54 \mathrm{a}$ & $0,73 \mathrm{a}$ & $0,75 \mathrm{a}$ \\
\hline Nemaguard & $2,77 \mathrm{a}$ & $3,17 \mathrm{a}$ & $0,29 \mathrm{a}$ & $0,26 \mathrm{a}$ & $1,30 \mathrm{a}$ & $2,32 \mathrm{a}$ & $2,31 \mathrm{a}$ & $2,03 \mathrm{~b}$ & $0,57 \mathrm{~b}$ & $0,60 \mathrm{~b}$ \\
\hline Okinawa & $2,60 \mathrm{a}$ & $3,08 \mathrm{a}$ & $0,27 \mathrm{a}$ & $0,27 \mathrm{a}$ & $0,88 \mathrm{~b}$ & $2,12 \mathrm{a}$ & $2,66 \mathrm{a}$ & $2,50 \mathrm{a}$ & $0,66 \mathrm{a}$ & $0,72 \mathrm{a}$ \\
\hline$F_{p c}$ & $1,00^{\text {ns }}$ & $1,74^{\mathrm{ns}}$ & $1,31^{\mathrm{ns}}$ & $0,51^{\mathrm{ns}}$ & $3,26^{*}$ & $2,43^{\mathrm{ns}}$ & $0,95^{\mathrm{ns}}$ & $10,44^{* *}$ & $9,61^{* *}$ & $28,77^{* *}$ \\
\hline $\mathrm{F}$ & $2,06^{\mathrm{ns}}$ & $0,54^{\mathrm{ns}}$ & $0,77^{\mathrm{ns}}$ & $0,30^{\mathrm{ns}}$ & $0,05^{\text {ns }}$ & $0,59^{\mathrm{ns}}$ & $0,23^{\mathrm{ns}}$ & $0,56^{\mathrm{ns}}$ & $4,21^{*}$ & $0,71^{\mathrm{ns}}$ \\
\hline CV $(\%)$ & 6,70 & 5,10 & 13,79 & 10,33 & 14,95 & 7,71 & 11,15 & 5,51 & 6,24 & 3,75 \\
\hline
\end{tabular}

Médias seguidas por letras distintas, na mesma coluna, diferem entre si, pelo teste de Tukey. " significativo ao nível de $5 \%$ de probabilidade; ${ }^{* *}$ significativo ao nível de $1 \%$ de probabilidade; ${ }^{\text {ns }}$ não significativo. Interpretação dos resultados da análise foliar do pessegueiro, segundo SBCS/CQFS (2004): $\square$ =abaixo do normal; $\square=$ normal; $\square$ = acima do normal. 
TABELA 3-Conteúdo de micronutrientes nas folhas $\left(\mathrm{em} \mathrm{mg} \mathrm{kg}^{-1}\right)$ da cv. Maciel de pessegueiro, em diferentes porta-enxertos, em dois pomares experimentais. Embrapa Clima Temperado, 2012-2013.

\begin{tabular}{|c|c|c|c|c|c|c|c|c|c|c|}
\hline \multirow{2}{*}{$\begin{array}{c}\text { Porta- } \\
\text { enxerto }\end{array}$} & & \multicolumn{2}{|c|}{ Mn } & \multicolumn{2}{|c|}{$\frac{Z n_{-}}{\sigma_{k 0}=}$} & \multicolumn{3}{|c|}{$\mathrm{Cu}_{-\ldots} \ldots \ldots$} & \multirow{2}{*}{2013} \\
\hline & 2012 & 2013 & 2012 & 2013 & 2012 & 201 & 2012 & 2013 & 2012 & \\
\hline & \multicolumn{10}{|c|}{ Pomar 1} \\
\hline Aldrighi & $92,5 \mathrm{a}$ & $82,8 \mathrm{a}$ & $105,8 \mathrm{a}$ & $133,0 \mathrm{a}$ & $13,3 \mathrm{a}$ & $14,0 \mathrm{ab}$ & $7,8 \mathrm{a}$ & $6,0 \mathrm{a}$ & $28,3 \mathrm{a}$ & $12,3 \mathrm{a}$ \\
\hline Capdeboscq & $103,0 \mathrm{a}$ & $83,8 \mathrm{a}$ & $87,8 \mathrm{a}$ & $111,8 \mathrm{a}$ & $14,0 \mathrm{a}$ & $13,5 \mathrm{~b}$ & $7,0 \mathrm{a}$ & $5,8 \mathrm{a}$ & $5,3 \mathrm{~b}$ & $12,5 \mathrm{a}$ \\
\hline Flordaguard & $98,3 \mathrm{a}$ & $86,0 \mathrm{a}$ & $78,5 \mathrm{a}$ & $105,3 \mathrm{a}$ & $15,5 \mathrm{a}$ & $14,5 \mathrm{ab}$ & $7,0 \mathrm{a}$ & $6,5 \mathrm{a}$ & $27,3 \mathrm{ab}$ & $12,8 \mathrm{a}$ \\
\hline Nemaguard & $98,0 \mathrm{a}$ & $80,5 \mathrm{a}$ & $74,3 \mathrm{a}$ & $107,8 \mathrm{a}$ & $14,0 \mathrm{a}$ & $14,8 \mathrm{ab}$ & $7,0 \mathrm{a}$ & $7,0 \mathrm{a}$ & $29,0 \mathrm{a}$ & $20,8 \mathrm{a}$ \\
\hline Okinawa & $104,3 \mathrm{a}$ & $88,3 \mathrm{a}$ & $88,5 \mathrm{a}$ & $129,3 \mathrm{a}$ & $14,5 \mathrm{a}$ & $15,8 \mathrm{a}$ & $6,3 \mathrm{a}$ & $6,5 \mathrm{a}$ & $29,0 \mathrm{a}$ & $20,8 \mathrm{a}$ \\
\hline $\mathrm{F}$ & $0,87^{\mathrm{ns}}$ & $0,76^{\mathrm{ns}}$ & $1,26^{\mathrm{ns}}$ & $2,47^{\mathrm{ns}}$ & $1,00^{\text {ns }}$ & $4,48^{*}$ & $0,67^{\mathrm{ns}}$ & $2,71^{\mathrm{ns}}$ & $8,17^{* *}$ & $1,22^{\mathrm{ns}}$ \\
\hline $\mathrm{F}$ & $4,03^{*}$ & $1,02^{\text {ns }}$ & $1,94^{\mathrm{ns}}$ & $13,23^{* *}$ & $7,73^{* *}$ & $9,25^{* *}$ & $1,02^{\mathrm{ns}}$ & $0,52^{\text {ns }}$ & $3,24^{\text {ns }}$ & $16,97^{* *}$ \\
\hline CV (\%) & 10,07 & 8,15 & 24,83 & 13,90 & 11,64 & 5,52 & 18,58 & 9,32 & 3,96 & 51,77 \\
\hline & \multicolumn{10}{|c|}{ Pomar 2} \\
\hline Aldrighi & $77,8 \mathrm{ab}$ & $83,5 \mathrm{a}$ & $37,8 \mathrm{a}$ & $59,0 \mathrm{a}$ & $6,8 \mathrm{a}$ & $7,5 \mathrm{a}$ & $6,0 \mathrm{~b}$ & $4,8 b$ & $26,5 \mathrm{a}$ & $12,5 \mathrm{a}$ \\
\hline Capd & & 85 & $35,0 \mathrm{a}$ & $53,0 \mathrm{a}$ & $6,3 \mathrm{a}$ & $7,8 \mathrm{a}$ & $6,5 \mathrm{ab}$ & $5,0 a b$ & $25,3 \mathrm{a}$ & $11,5 \mathrm{a}$ \\
\hline Flordaguard & $80,8 \mathrm{ab}$ & $86,8 \mathrm{a}$ & $31,0 \mathrm{a}$ & $40,5 \mathrm{a}$ & $8,5 \mathrm{a}$ & $7,5 \mathrm{a}$ & $7,5 \mathrm{a}$ & $5,8 \mathrm{a}$ & $26,5 \mathrm{a}$ & $11,5 \mathrm{a}$ \\
\hline Nemaguard & $72,5 \mathrm{~b}$ & $82,5 \mathrm{a}$ & $31,5 \mathrm{a}$ & $61,0 \mathrm{a}$ & $6,3 \mathrm{a}$ & $7,3 \mathrm{a}$ & $6,5 \mathrm{ab}$ & $4,8 \mathrm{~b}$ & $27,5 \mathrm{a}$ & $12,3 \mathrm{a}$ \\
\hline Okinawa & $78,8 \mathrm{ab}$ & $81,5 \mathrm{a}$ & $35,0 \mathrm{a}$ & $49,5 \mathrm{a}$ & $8,5 \mathrm{a}$ & $8,5 \mathrm{a}$ & $6,3 \mathrm{~b}$ & $5,0 \mathrm{ab}$ & $26,0 \mathrm{a}$ & $13,0 \mathrm{a}$ \\
\hline $\mathrm{F}$ & $4,27^{*}$ & $0,29^{\text {ns }}$ & $1,22^{\mathrm{ns}}$ & $2,44^{\text {ns }}$ & $4,57^{*}$ & $0,93^{\text {ns }}$ & $4,88^{*}$ & $4,76^{*}$ & $1,29^{\text {ns }}$ & $1,24^{\mathrm{ns}}$ \\
\hline $\mathrm{F}$ & $3,84^{*}$ & $2,36^{\text {ns }}$ & $1,63^{\text {ns }}$ & $3,23^{\text {ns }}$ & $13,09^{*}$ & $0,20^{\text {ns }}$ & $3,19^{\mathrm{ns}}$ & $1,29^{\mathrm{ns}}$ & $1,04^{\mathrm{ns}}$ & $864,8^{* *}$ \\
\hline $\mathrm{CV}$ & 7,17 & 14,77 & 17,43 & 19,92 & 14,95 & 12,93 & 7,88 & 7,45 & 5,50 & 9,62 \\
\hline
\end{tabular}

Médias seguidas por letras distintas, na mesma coluna, diferem entre si, pelo teste de Tukey. * significativo ao nível de $5 \%$ de probabilidade; ** significativo ao nível de $1 \%$ de probabilidade; ns não significativo. Interpretação dos resultados da análise foliar do pessegueiro, segundo SBCS/CQFS (2004):

$\square$ = abaixo do normal; $\square$ normal;

\section{CONCLUSÕES}

Os porta-enxertos 'Aldrighi', 'Capdeboscq', 'Flordaguard', 'Nemaguard' e 'Okinawa' não influenciaram nos teores foliares de $\mathrm{P}$ e de $\mathrm{Mn}$ da cv. Maciel, em ambos os pomares e anos de avaliação.

O porta-enxerto 'Nemaguard' reduz nos teores foliares de $\mathrm{Mg}$ da $\mathrm{cv}$. Maciel, sem alterar a classe de interpretação agronômica.

Embora os teores foliares de N, Fe, Zn e B tenham sido abaixo do normal ou insuficientes, em praticamente todas as amostras de ambos os pomares e anos de avaliação, os porta-enxertos testados não influenciaram nestas interpretações.

\section{AGRADECIMENTOS}

Os autores agradecem ao CNPq e à Embrapa, pelo suporte financeiro; aos extensionistas da Emater Escritório Municipal de Pelotas, ao técnico agrícola Rudinei Oliveira Gomes e ao persicultor Fábio Donini, pelo apoio na condução deste trabalho.

\section{REFERÊNCIAS}

BARBOSA, W.; CAMPO DALL'ORTO, F.A.; OJIMA, M.; MARTINS, F.P. Produção e manejo de sementes do pessegueiro porta-enxerto Okinawa. $\mathbf{O}$ Agronômico, Campinas, v.45, n.2/3, p.10-16, 1993.

BOYHAN, G.E.; NORTON, J.D.; PITTS, J.A. Establishment, growth, and foliar nutrient content of plum trees on various rootstocks. HortScience, Alexandria, v.30, n.2, p.219-221, 1995.

BROWN, S.K.; CUMMINS, J.N. Rootstock effect on foliar nutrient concentrations of 'Redhaven' peach trees. HortScience, Alexandria, v.24, n.5, p.769-771, 1989.

BROOKS, R.M.; OLMO, H.P. Register of new fruit and nut varieties. Nemaguard peach. Proceedings of the American Society for Horticultural Science, Mount Vernon, v.78, p.634-635, 1961.

CAMPO DALL'ORTO, F.A.; OJIMA, M.; BARBOSA, W.; MARTINS, F.P. O nanismo do pessegueiro induzido pela enxertia no damasqueirojaponês. Pesquisa Agropecuária Brasileira, Brasília, v.27, n.3, p.517-521, 1992. 
COUVILLON, G.A. Leaf elemental content comparisons of own-rooted peach cultivars to the same cultivars on several peach seedling rootstocks. Journal of the American Society for Horticultural Science, Mount Vernon, v.107, n.4, p.555-558, 1982.

DE ROSSI, A.; FACHINELLO, J.C.; RUFATO, L.; PARISOTTO, E.; PICOLOTTO, P.; KRUGER, L.R. Comportamento do pessegueiro 'Granada' sobre diferentes porta-enxertos. Revista Brasileira de Fruticultura, Jaboticabal, v.26, n.3, p.446-449, 2004.

ESTAT. Sistema para análises estatísticas (v. 2.0). Jaboticabal: Departamento de Ciências Exatas, FCAV-UNESP, 1994.

FREIRE, C.J.S. Recomendação de adubação potássica de manutenção para a cultura da ameixeira por meio da análise foliar. Pelotas: Embrapa Clima Temperado, 2002.2p. (Comunicado Técnico, 67).

FREIRE, C.J.S.; MAGNANI, M. Manual de coleta de amostras de folhas, para diagnose nutricional, das principais frutíferas cultivadas no RS e em SC. Pelotas: Embrapa Clima Temperado, 2005. 18p. (Documentos, 142).

GALARÇA, S.P.; FACHINELLO, J.C.; BETEMPS, D.L.; MACHADO, N.P.; HAAS, L.B.; PRESOTTO, M.E.; COMIOTTO, A. Produção e qualidade de frutos de pessegueiros 'Chimarrita' e 'Maciel' sobre diferentes porta-enxertos. Pesquisa Agropecuária Brasileira, Brasília, v.47, n.12, p.1731-1736, 2012.

JOHNSON, R.S.; ANDRIS, H.; DAY, K.; BEEDE, R. Using dormant shoots to determine the nutritional status of peach trees. Acta Horticulturae, The Hague, n.721, p.285-290, 2006.

JOHNSON, R.S. Nutrient and water requirements of peach trees. In: LAYNE, D.; BASSI, D. The peach: botany, production and uses. Wallingford: CABI, 2008. p.303-331.

KNOWLES, J.W.; DOZIER JR., W.A.; EVANS, C.E.; CARLTON, C.C.; MCGUIRE, J.M. Peach rootstock influence on foliar and dormant stem nutrient content. Journal of the American Society for Horticultural Science, Mount Vernon, v.109, n.3, p.440-444, 1984.
LEONEL, S.; SOUZA, M.E.; TECCHIO, M.A.; SEGANTINI, D.M. Leaf nutritional levels in peach and nectarine grown in subtropical climate. Revista Brasileira de Fruticultura, Jaboticabal, v.33, p.752761, 2011. Número especial.

MAYER, N.A.; PEREIRA, F.M.; KOBA, V.Y. Desenvolvimento inicial no campo de pessegueiros 'Aurora-1' enxertados em clones de umezeiro e 'Okinawa' propagados por estacas herbáceas. Revista Brasileira de Fruticultura, Jaboticabal, v.28, n.2, p.231-235, 2006.

PEREIRA, F.M.; MAYER, N.A. Pessegueiro: tecnologias para a produção de mudas. Jaboticabal: Funep, 2005. 65p.

RASEIRA, M.C.B., NAKASU, B.H. Cultivares: descrição e recomendação. In: MEDEIROS, C.A.B., RASEIRA, M.C.B. A cultura do pessegueiro. Brasília: Embrapa-SPI; Pelotas: Embrapa-CPACT, 1998. p.29-99.

RASEIRA, A.; PEREIRA, J.F.M.; MEDEIROS, A.R.M.; CARVALHO, F.L.C. Instalação e manejo do pomar. In: MEDEIROS, C.A.B., RASEIRA, M.C.B. A cultura do pessegueiro. Brasília: Embrapa-SPI; Pelotas: Embrapa-CPACT, 1998. p.130-160.

REIGHARD, G.L.; BRIDGES, W.; RAUH, B.; MAYER, N.A. Prunus rootstocks influence peach leaf and fruit nutrient content. Acta Horticulturae, The Hague, n.984, p.117-124, 2013.

ROMBOLÀ, A.D.; SORRENTI, G.; MARODIN, G.A.B.; DE PIERI, A.Z.; BARCA, E. Nutrição e manejo do solo em fruteiras de caroço em regiões de clima temperado. Semina: Ciências Agrárias, Londrina, v.33, n.2, p.639-654, 2012.

ROMERA, F.J.; ALCÁNTARA, E.; LA GUARDIA, M.D. Characterization of the tolerance to iron chlorosis in different peach rootstocks grown in nutrient solution. Plant and Soil, The Hague, n.130, p.115-119, 1991.

SHERMAN, W.R.; LYRENE, P.M.; SHARPE, R.H. Flordaguard peach rootstock. HortScience, Alexandria, v.26, n.4, p.427-428, 1991. 
SBCS/CQFS - Sociedade Brasileira de Ciências do Solo; Comissão de Química e Fertilidade do Solo. Manual de adubação e de calagem para os Estados do Rio Grande do Sul e de Santa Catarina. 10.ed. Porto Alegre: Sociedade Brasileira de Ciência do Solo/Núcleo Regional Sul, 2004. 400 p.

TAGLIAVINI, M.; ZAVALLONI, C.; ROMBOLA, A.D.; QUARTIERI, M.; MALAGUTI, D.; MAZZANTI, F.; MILLARD, P.; MARANGONI, B. Mineral nutrient partitioning to fruits of deciduous trees. Acta Horticulturae, The Hague, n.512, p.131$140,2000$.

TSIPOURIDIS，C.G.; SIMONIS，A.D.;
BLADENOPOULOS, S., ISAAKIDIS, A.M.; STYLIANIDIS, D.C. Nutrient element variability of peach trees and tree mortality in relation to cultivars and rootstocks. Horticultural Science, Prague, v.29, n.2, p.51-55, 2002.

ZARROUK, O.; GOGORCENA, Y.; GÓMEZAPARISI, J.; BETRÁN, J.A.; MORENO, M.A. Influence of almond $\mathrm{x}$ peach hybrids rootstocks on flower and leaf mineral concentration, yield and vigour of two peach cultivars. Scientia Horticulturae, Amsterdam, n.106, p.502-514, 2005. 